\title{
Removal of Artifacts from EEG Signals: A Review
}

\author{
Xiao Jiang ${ }^{1,2}$, Gui-Bin Bian ${ }^{1, * \mathbb{C}}$ and Zean Tian ${ }^{2}$ \\ 1 Institute of Automation, Chinese Academy of Science, Beijing 100190, China; jx19950427@163.com \\ 2 School of Big Data and Information Engineering, Guizhou University, Guiyang 550025, China; \\ tianzean@126.com \\ * Correspondence: guibin.bian@ia.ac.cn
}

Received: 15 December 2018; Accepted: 21 February 2019; Published: 26 February 2019

\begin{abstract}
Electroencephalogram (EEG) plays an important role in identifying brain activity and behavior. However, the recorded electrical activity always be contaminated with artifacts and then affect the analysis of EEG signal. Hence, it is essential to develop methods to effectively detect and extract the clean EEG data during encephalogram recordings. Several methods have been proposed to remove artifacts, but the research on artifact removal continues to be an open problem. This paper tends to review the current artifact removal of various contaminations. We first discuss the characteristics of EEG data and the types of different artifacts. Then, a general overview of the state-of-the-art methods and their detail analysis are presented. Lastly, a comparative analysis is provided for choosing a suitable methods according to particular application.
\end{abstract}

Keywords: electroencephalogram; artifact removal techniques; artifacts

\section{Introduction}

With the emergence of non-invasive techniques, the research of neuroscience, cognitive science and cognitive psychology has been developed by electroencephalograph (EEG), functional near-infrared spectroscopy (fNIRS), magnetoencephalography (EMG) and other key tools [1]. EEG is one of the tools used for analyzing brain activity, which signals can be recorded from several electrodes on the scalp [2]. As a special and complicated biological electricity signal, it reflects the functional state of brain allied to the person's mental condition, from which we can extract the vital information, and further monitor patient's heath [3], diagnosis and identify different brain conditions [4,5]. However, EEG is high temporal resolution and its signals are easily contaminated by undesired noise, which will resulting in various artifacts [6]. The cause of artifacts may arise from measurement instrument and human subjects, the prior one including faulty electrodes, line noise and high electrode impedance [7], which can be avoided by more precise recording system and strictly recording procedures, whereas physiological artifacts are more complicated to remove. For instance, eye movements, eye blinks, cardiac activity and muscle activity occurred in EEG signal are some major types of physiological artifacts [8].

Such physiological artifacts may interfere with neural information and even be used as normal phenomena to misleadingly drive a practical application such as brain-computer interface [9]. Furthermore, artifacts might also imitate cognitive or pathologic activity and therefore bias the visual interpretation and diagnosis in clinical research such as sleep order, Alzheimer's disease [10,11], etc. Therefore, the requirement of artifacts identification and removal, either in clinical diagnosis or practical applications, are the most important preprocessing step prior to be utilized. One straightforward way is to apply precautions to avoid unnecessary motion incurring artifacts, but it will get into trouble if subjects have inability to follow such extra instruction, and furthermore this method is unwieldy either for clinical or household applications. Instead of artifact avoidance, manual segment rejection directly omit epochs that contaminated by artifacts. Consequently, this method will significantly 
loss possibly useful neural signals. To this end, a variety of efficient techniques for artifact removal, especially for physiological artifacts, has been proposed in the published literatures. Such published techniques advanced by improving existing algorithms, combining various methods or making removal process automatic can be primarily separated into two categories: either by estimation of the artifactual signals using reference channel or by decomposing the EEG signal into other domains. Alternatively, these techniques range from Regression [12], Blind Source Separation (BSS) [13-15], Empirical-mode Decomposition (EMD), Wavelet Transform algorithm to their hybrid methods [16]. To obtain a comprehensive overview of EEG artifact removal techniques developed in different studies, we used Google Scholar as the main search engine. The keywords were "electroencephalogram", "EEG artifacts", "denoising", "artifact correction" and "artifact removal method". Boolean operators (AND, OR, and NOT) were used to combine search terms. Figure 1 provides the chart that percentage of the number of literatures in the past five years. It indicate that the BSS-based methods, especially ICA, are the most commonly use algorithms. Also it is worthy to note that from the chart, due to the limits of single method like Regression and BSS, many researchers tend to prefer the hybrid method to enhance the performance of techniques in recent years. Despite the extensive research centered on artifact detection and removal of EEG signals reported in the literature to date, there is no consensus of one optimal solution for all types of artifacts.

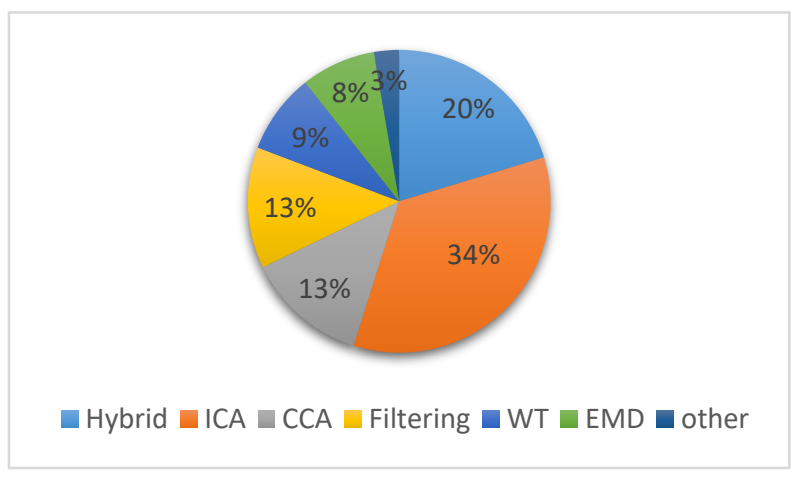

Figure 1. Percentage of the number of references published during the past three years.

Considering this issue, we attempt to do a comprehensive review of main methods proposed in literature for artifact removal on EEG. We firstly review the characteristic of EEG signal and the types of artifacts existed. Then, we present the widely applied removal techniques and their advantage and drawbacks. Finally, a comparative analysis according to particular requirement is discussed. We believe that the knowledge provided in this review can help to determine a removal technique, which satisfies the necessary requirements for a particular application.

\section{Background}

\subsection{Characteristics of the EEG}

EEG is the recording of the brain's spontaneous electrical activity and provide the measurement Voltage fluctuations of brain activity $[17,18]$. The frequency of EEG signals range from $0.01 \mathrm{~Hz}$ to around $100 \mathrm{~Hz}$, which can be divided into five frequency bands, and four basic types are summarized in Table 1.

Table 1. Basic brain wave with their frequency.

\begin{tabular}{ccc}
\hline Band Name & Frequency $\mathbf{( H z )}$ & Interpretation \\
\hline Delta & $<4$ & Deep sleep \\
Theta & $4-8$ & Relaxed state and meditation \\
Alpha & $8-13$ & Relaxed state of consciousness \\
Beta & $13-30$ & active thinking \\
\hline
\end{tabular}




\subsection{Types of Artifacts}

Signal artifacts are more significant while collecting EEG data from recording systems $[19,20]$. These artifacts can contaminate the quality of EEG data. In this regard, a comprehensive knowledge of the types of artifacts is requisite to remove the artifacts or noise efficiently. Artifacts are unwanted signals which are mainly originated from environment noise, experimental error and physiological artifacts. Furthermore, the environment artifacts and experiment error, which come from external factors, are classified as extrinsic artifacts, whereas the physiological from body itself (e.g., eye blink, muscle activity, heart beat) can be categorized as intrinsic artifacts [21-23]. Figure 2 shows the three major physiological artifacts in the literature. The environment artifacts can be eliminated by a simple filter due to the frequency of such artifacts are inconsistent with desired signals [21]. Proper procedure and planning can reduced experimental error easily [19]. Nevertheless, the physiological artifacts are more difficult to be removed as they require particular algorithms [24]. We next discuss the major physiological artifacts affecting EEG data.

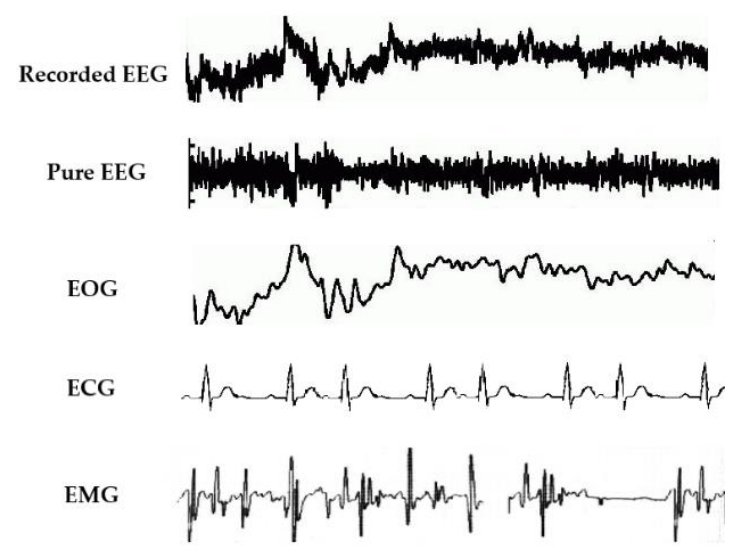

Figure 2. Physiological artifacts present in EEG signals.

\subsubsection{Ocular Artifacts}

Ocular artifacts generate significant artifacts in the EEG recordings [25]. The origin of ocular artifacts is eye movement and blinks which can propagate over the scalp and be recorded by EEG activity. More specifically, eye movement artifacts produce by changes in orientation of the retina and cornea dipole, and blink artifacts caused by ocular conductance due to the alterations of contact of the cornea with eyelid [26]. In addition, because of volume conduction effect, both ocular artifact and EEG activity propagated to head surface and record by the electrodes. Such ocular signals can be recorded using electrooculogram (EOG). The amplitude of EOG is generally many times greater than EEG [25-27] and its frequency are similar with the frequency of EEG signals. Worthy to note that not only EEG data can be contaminated by the EOG, but in turn, EOG can also be contaminated by EEG. Consequently, a bidirectional interference will introduce removal error when we remove EOG artifacts [28].

\subsubsection{Muscle Artifacts}

Contamination of EEG data by muscle activity is a well-recognized tough problem as it arises from different type of muscle groups [27,29]. These artifacts can be caused by any muscle proximity to signal recording sites contraction and stretch, the subject talks, sniffs, swallows [21], etc. In theory, muscle artifacts measured by electromyogram (EMG) have a broad distribution from $0 \mathrm{~Hz}$ to $>200 \mathrm{~Hz}[21,30]$. The degree of muscle contraction and stretch will affect the amplitude and waveform of artifacts. It is extremely difficult to obtain the activity from a single channel measurement comparing to EOG and eye-tracking. Therefore, EMG artifacts are particularly challenging to eliminate. Additionally, EMG contamination and EEG have substantial statistical independence from each other both temporally 
and spatially. This imply that the Independent Components Analysis might be a suitable methods to remove EMG contamination [31,32].

\subsubsection{Cardiac artifacts}

Cardiac artifacts can be introduced when the electrodes is placed on or near a blood vessel [29], in which the movement of expansion and contraction due to the heart. Such artifacts called pulse artifacts, whose frequency is around $1.2 \mathrm{~Hz}$, can occur within EEG as a similar waveform, hence it is hard to remove [27]. Another cardiac activity known as ECG measure the electrical signal outputted from the heart [33]. In contrast to pulse artifacts, ECG can be measured with characteristic regular pattern, and be recorded aside cerebral activity, hence removing such artifacts may be easier just using a reference waveform.

\subsubsection{Extrinsic Artifacts}

In addition to the artefacts mentioned above, external source of artifacts also have a damaging effect on EEG measurement. Instrument artifacts as a type of extrinsic artifacts originate from the electrode misplacement and cable movements. These artifacts can be removed by appropriate procedure and planning. The electromagnetic interference emitted from surroundings is another type external artifact that affect the EEG recordings. Such artifacts from environmental sources can be easily degraded by a simple filter due to its distinguishable frequency band. Despite the broad frequency band of white noise, a high-frequency filter still can remove majority of artifacts [13]. Since the activity of brain area can be observed in many channels, the coherence between EEG channels will introduce volume conduct artefact [34]. Relevant literature to deal with volume conduct artifact can be found in $[35,36]$.

\section{Single Artifacts Removal Techniques}

\subsection{Regression Methods}

The traditional method for removing artifacts from EEG is the regression methods [37]. It is applied under the assumption that each channel is the cumulative sum of pure EEG data and a proportion of artifact [13]. Regression analysis first defines the amplitude relation between reference channel and EEG channel by transmission factors, and then subtracting the estimated artifacts from EEG. Thus, this algorithm requires exogenous reference channels (i.e., EOG, ECG) to omit different artifacts. When dealing with ocular activity, the EEG data can be obtained as:

$$
\mathrm{EEG}_{\mathrm{cor}}=\mathrm{EEG}_{\mathrm{raw}}-\gamma \mathrm{F}(\mathrm{HEOG})-\delta \mathrm{F}(\mathrm{VEOG})
$$

where $\gamma$ and $\delta$ depend on the transmission coefficient between EOG and EEG, and EEG cor $_{\text {and EEG }}$ raw represent corrected EEG data and raw EEG data, respectively. HEOG and VEOG denote the recordings from horizontal and vertical EOG channels.

Hillyard and Gallambos [38] firstly proposed approaches based on time domain regression to remove ocular activity. Whitton et al. [39] introduced the frequency domain regression and combined this method with EEG detection software accordingly. However, either in time-domain or frequency-domain, such regression approaches are affected by bidirectional methods, [25]. This is due to ocular potentials contaminate EEG data, similarly the EEG data can contaminate ocular recording. To this end, Wallstrom et al. used a filtering prior to applying Bayesian adaptive regression splines $[40,41]$. In doing so, the issues of bidirectional contamination is substantially reduced.

Although the simplified model and reduced computational demands of regression methods, the need for one or more good regression reference channels limit their capacity for removing EOG and ECG [21]. Beyond that, anastomotic regression channels to eliminate each muscle artifact are not 
always available. Nevertheless, despite researches prefer blind source separation-based methods, regression-based algorithms are still the gold standard to assess the performance of new approach.

\subsection{Wavelet Transform}

Wavelet transform, transforming a time domain signal into time and frequency domain, has good time-frequency features relative to Fourier transform due to the better tunable time-frequency tradeoff and superiority of non-stationary signal analysis [42]. The transformation is accomplished by selecting the subsets of the scales ' $j$ ' and the time shift ' $k$ ' of the mother wavelet $\psi(t)$. Mathematically:

$$
\psi_{\mathrm{j}, \mathrm{k}}(\mathrm{t})=2^{\frac{\mathrm{j}}{2}} \psi\left(2^{\mathrm{j}} \mathrm{t}-\mathrm{k}\right),
$$

where $\mathrm{j}$ and $\mathrm{k}$ are integers. Then the wavelet transform can be performed by:

$$
\mathrm{W}_{\psi}=<\mathrm{f}, \psi_{\mathrm{j}, \mathrm{k}}>,
$$

which means the inner product of time-domain signal and wavelet function.

The DWT (discreted wavelet transform) derived from continuous wavelet can be applied when the input signal, and the decomposition can be expressed as:

$$
\begin{gathered}
X_{a, L}[n]=\sum_{k=1}^{N} X_{a-1, L}[2 n-k] g[k], \\
X_{a, H}[n]=\sum_{k=1}^{N} X_{a-1, L}[2 n-k] h[k],
\end{gathered}
$$

where $g[n]$ is the low pass filter to generate low frequency component and $h[n]$ is high pass filter to get high frequency component. After the decomposition of EEG data using wavelet transformation, thresholding is applied to discard the signal that contain artifacts. The remaining details are added up to reconstruct the clean signal [43]. Even though its versatility of artifact attenuation, the DWT fails to identify artifacts completely that overlap with the spectral properties, hence recent work prefer the combination of DWT with other methods, like ICA.

\subsection{BSS}

The BSS method includes a variety of unsupervised learning algorithms without prior information and extra reference channels. The general methodology of BSS can be described as follows. Let X be observed signals obtained from scalp electrodes. Also, let $S$ be the source signals which includes original signals and artifacts. These source signals are linear mixed by an unknown matrix A:

$$
\mathrm{X}=\mathrm{AS},
$$

to get the observed signals. The BSS algorithm is a reversed version:

$$
\mathrm{U}=\mathrm{WX}
$$

where $\mathrm{U}$ is the estimation of sources and the $\mathrm{W}$ is the reverse mixing of $\mathrm{X}$. Then components representing the artifacts are removed and the remaining components reconstruct EEG data to achieve the purpose of denoising. In that following, we describe some representative works that have adopted the BSS algorithms.

\subsubsection{Principal Component Analysis}

PCA is one of the simplest and widely used BSS techniques, which algorithm is based on Eigen values of covariance matrix [44]. In this method, it firstly converts correlated variables into 
uncorrelated variables using orthogonal transformation. Such uncorrelated variables are called principal components (PCs). These PCs of EEG signals will be implemented using Single Value Decomposition (SVD).

Berg and Scherg [45] firstly introduced principal component analysis to remove ocular artifacts. In this work, the major components representing blinks and eye movements are extracted by PCA of variance. Then the artifact-free EEG data was obtained by rejecting the related components through an inverse operation. Casarotto et al. [46] demonstrated that PCA perform more computationally efficient than linear regression methods. However, the requirement that artifact components are uncorrelated with EEG data is generally hard to satisfy. Besides of that, PCA fails to separate the interferences when the potential of drifts and EEG data are similar. Consequently, the subsequent research prefer other flexible methods such as ICA.

\subsubsection{Independent Component Analysis}

Another method known as ICA, assuming that signal sources are instantaneously linear mixtures of cerebral and artifactual sources, can decompose observed signal into independent components (ICs) [14]. Once ICs are extracted from original signals, the clean signal reconstructed by discarding ICs contained artifacts.

Considering that the simple network algorithm method achieved by Bell and Sejnowski blindly separate signals using information maximization, Makeig et al. firstly applied the ICA algorithm to analyze EEG and EPR signals [47]. In contrast to traditional artifact cancelling approaches, Vigaro et al. tested the ICA method on simulated and experimental data, and showed good performance in the separation of signals from their linear mixtures and extraction of the eye information present in EOG signals [48,49]. In 2000, Jung et al. removed artifacts from EEG by the extended ICA, and results comparing effectively to regression algorithm [50]. Romero et al. applied ICA to reduce EEG artifacts in different sleep stages, and found the bidirectional property of EEG and EOG had little effect on ICA [51]. A method using probability and kurtosis to eliminate semi-automatically was discussed by Delorme et al. [52]. In order to avoid the errors introduced by manually select components, Joyce et al. [53] introduced an automatic extraction and removal of eye movement artifacts after the ICA analysis. In recent years, the study on automatic removal of artifact based on ICA has been developed by [54-58]. Frølich and Dowding compared five commonly used variants of ICA methods suitable for oscillatory activity. And the author find that the adequately high-pass filtering is very important and the extended Infomax perform best [59].

As an extension of PCA which be constrained to transform directions that are orthogonal, ICA has been proven more effective and flexible in source separation of EEG signals form artifacts under the premise below:

(1) Source signals are statistically independent from each other and instantaneously mixed.

(2) The dimension of observation signal must be greater than or equal to source signal $[25,60]$.

(3) Sources are non-Gaussian or only one source are Gaussian.

In general, all artifacts like eye movements, eye blinks, cardio activities are generated by mutually independent source and volume conduction is linear, which makes the first premise is reasonable. However, the acquisition of biomedical signal is usually not linear instantaneous, and therefore, a Convolutive ICA (CICA) approach considering weighted and delayed contributions of signals was proposed in [61]. Additionally, ICA can estimate original signals which are non-Gaussian, but unfortunately sources are usually not known to be Gaussian or non-Gaussian. Another problem is that the scalp EEG are the result of the joint activity from multiple neurons, therefore, separating the source signals when the number of sources is greater than the number of sensors is promising. 


\subsubsection{Canonical Correlation Analysis}

Canonical correlation analysis (CCA) is another commonly employed BSS technique. Unlike ICA method that use higher order statistics, CCA use second order statistics which bring shorter computational time. CCA also differ from ICA in its conditions to separate components. The CCA separate components from uncorrelated sources whereas ICA from statistical independent sources [62]. CCA find the linear relation between two multi-dimensional random variables by maximize the pairwise correlations across the two data sets. [63]. In contrast to ICA that only takes statistical distribution of the same sample values into account, CCA consider the autocorrelation in the source signals and demonstrated that have similar qualitative results, but consume a little computational complexity. The CCA has been applied to remove muscle artifacts from EEG signals has been first discussed in [64]. CCA has also been used in the removal of muscle artifacts from EEG recordings of spoken language production [65]. The author investigated the difference in autocorrelation between brain and muscle artifacts. Then the components with least autocorrelation were selected and removed. In order to further explore potential nonlinear process, an eigenspace maximal information canonical correlation analysis (emiCCA) framework was proposed in [65]. Due to the form of muscle activity, which do not have stereotyped topography, the CCA algorithms outperform ICA to remove muscle artifacts.

\subsubsection{Source Imaging Based Method}

EEG source imaging (ESI) is a model-based imaging technique that combines temporal and spatial components of EEG to find the source of scalp-recorded potentials [66]. There are two fundamental issues in ESI: forward and inverse problem. The equivalent distributed dipole layer inversion from scalp recordings is a linear inversion that can be solved by minimum norm (MN). In current practice, various regularizations or weighted MN solutions are practically utilized [67]. Then the equivalent distributed source from EEG recordings can be reconstructed. Next, the first component discarded after the analysis of PCA, remaining components can be reconstructed the artifact-free EEG [68,69].

\subsection{Empirical Mode Decomposition}

Empirical mode decomposition (EMD) was first discussed in 1998 [70] as a heuristic technique for non-stationary and non-linear signal processing. EMD algorithm decomposes the signal, $x[n]$, into a set of components with amplitude-frequency modulated, $b[n]$, called intrinsic mode functions (IMFs) [71,72]. In the whole data set and all point, every IMF must be satisfies that the number of extrema are same with the number of zero crossings or differ at most by one, and the mean value of the envelope defined by the maxima and minima must be zero. Therefore, the EMD technique is empirical and data driven technique, whereas other methods depend on the selections of basic functions, such as wavelet analysis [73]. A sifting procedure is taken to calculate the IMF of a given signals and the steps shown below:

(1) Set $b[n]$ equal to input signal sequence $x[n]$.

(2) Calculate all the local maxima and local minima, and connect them separately with cubic spline interpolation. The upper envelope $\mathrm{u}[\mathrm{n}]$ and lower envelop $1[\mathrm{n}]$ are obtained.

(3) Calculate the mean value as: $\mu[n]=(\mathrm{u}[\mathrm{n}]+1[\mathrm{n}]) / 2$, and subtract it form original.

(4) Decide whether $\mathrm{b}[\mathrm{n}]$ is an IMF or not according to the condition described above.

(5) Repeat steps $2-4$ process until an IMF is obtained and assign $b[n]$ to $b_{k}[n]$.

(6) Once a IMF is obtained, generate the residue $r[n]$ as: $r[n]=r[n]-b_{k}[n]$.

(7) Repeat steps $1-5$ on the residue as the input signal sequence until the final residue is a constant, a monotonic function, or a function with only one maximum and one minimum.

Then the original signal can he reconstructed by:

$$
x[n]=\sum_{k=1}^{m} b_{k}(n)+r(n),
$$


where $r(n)$ represent the final residue signal [74]. Once the IMF determined, the artifact components of EEG data can be reflected, and then selected and removed. Finally, the pure EEG signal can be reconstructed by the newly IMFs. One drawback of EMD algorithm is that the sensitivity of noise, which incurring mode mixing complications [75]. The details about enhanced-EMD (EEMD) algorithm are discussed in [76], in which an average of a number of ensembles of EMD was utilized as the optimal IMFs, as a result, the robustness of EMD was improved. In some case, the reconstructed remaining IMFs can be entered to an extra artifact removal environment to enhance the quality of EEG data [77]. Another modified EMD, MEMD, also be proposed to simultaneously decompose multivariate signals into multivariate IMFs. Due to the simultaneously analysis of intrinsic modes across multiple channel, MEMD can more efficiently and accurately to remove artifacts, especially for broadband muscle artifacts [78].

\subsection{Filtering Methods}

Numerous filtering methods was employed in the cancelation of artifacts from the EEG, for instance, adaptive filtering, wiener filtering and Bayes filtering, in which different methods implemented with different principle of optimization [79]. Nevertheless, for the intention to minimize the mean square error between the predicted EEG and primary EEG, a weighting coefficient $\mathrm{W}$ will be adapted. Following article briefly illustrates two commonly used filtering approaches accordingly.

\subsubsection{Adaptive Filtering}

The underlying mechanism of adaptive filtering is to quantize the amount of artifactual contamination in the primary input, by iteratively adjusting the weights according the optimization algorithm, and subtract it from EEG with artifacts signals [80]. An illustration of the adaptive filtering is presented in Figure 3. The primary input is modelled as a mixture of clean and pure EEG data and an artifact source with the formula:

$$
\operatorname{EEG}_{\text {pri }}(\mathrm{n})=\mathrm{EEG}_{\text {pure }}(\mathrm{n})+\mathrm{N}(\mathrm{n}) \text {, }
$$

where $E E G_{\text {pri }}$ and $E E G_{\text {pure }}$ represent the primary signal and desired signal, respectively, and $\mathrm{N}$ represent noise signal which is an EOG artifact or an ECG interference according to the artifact to remove. Reference channel is given as one of the input to the filter. In order to obtain the pure

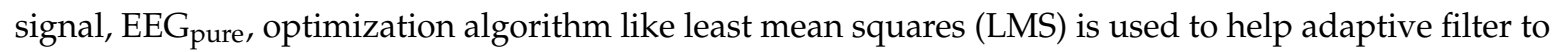
upgrade its weight parameter. Another alternative optimization algorithm is recursive least squares algorithm (RLMS) which convergence faster than LMS, but still requiring high calculation cost [81]. Disadvantages are that additional sensors is needed to provide reference inputs [82].

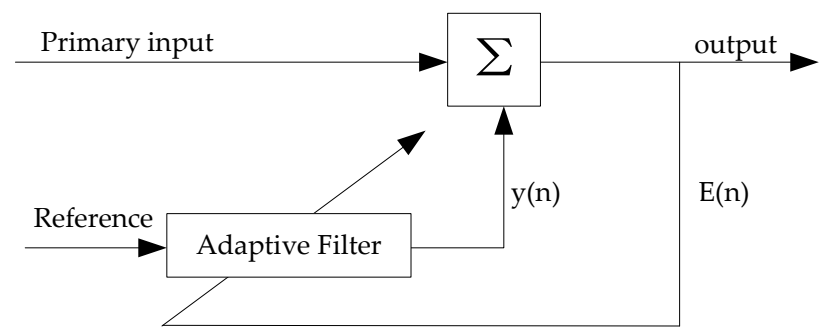

Figure 3. Functional diagram of an adaptive filter system.

\subsubsection{Wiener Filtering}

The wiener filtering is also an optimal filtering as the adaptive filtering, however, wiener filter technique is a linear statistical filtering technique used to estimating the true EEG data with the purpose to develop a linear time invariant filter to minimize the mean square error between the pure EEG data and the estimated signal [83]. The linear filter is found by estimating the power spectral densities of 
the measured signal and the artifact signal, since there is no prior knowledge on the statistics [84]. Although wiener filtering eliminate the limitation of extra reference, but the requirement of calibration will add the complexity of its application.

\subsection{Sparse Decomposition Methods}

Sparse component analysis (SCA) is another effect signal processing method to decompose signals sparsely in over-completer dictionary [85]. The over-complete dictionary can be calculate from complete dictionary by over-sampling. A dictionary can be constructed by waveforms or atoms, such as wavelet, Fourier and Dirac basis. After over-sampling, the orthogonality may not be true whereas the basis in the complete dictionary is orthogonal to each other. Signal sparsity can be measured with $l_{p}(0 \leq p \leq 1)$ as follows:

$$
\|x\|_{p}=\left(\sum_{j=1}^{n}\left|x_{j}\right|^{p}\right)^{\frac{1}{p}}
$$

where $X$ represents the signal, and $n$ is the dimension of $X$ [86]. Mallat and Zhang introduced a matching pursuit (MP) algorithm that decompose a signal into sparse representation [87]. The sparse decomposition of signal corresponding dictionary is obtained after several iterations, and each iteration choose a certain waveform with maximum inner product between itself and residual signal as the optimal one. A matching pursuit can isolate the signal structures that coherent with respect to a given dictionary. A modified MP method termed two dictionaries MP (TDMP) was proposed in to decompose signal more sparsely, and the decomposition results certify that TDMP are superior to MP [88]. In EEG analysis, Li et al. combined $\mathrm{L}_{1}$ norm-based Eigen decomposition into Common Spatial Patterns, and it effectively improve the robustness of BCI system to EEG outliers [89]. Another work developed a novel auto-regressive object function constructed in Lp norm space to compress the artifacts on EEG analysis [90].

\section{Hybrid Methods}

Beside from the algorithms concluded above, there are still many efficient and innovative methods. To exploit the advantage of each method, recently, several researchers have opted to use a hybrid strategy, which is a combination of two or more methods. Some major hybrid methods are discussed below.

\subsection{EMD-BSS}

In conjunction with ICA, these intrinsic mode functions (IMFs) are decomposed by an EMD or EEMD algorithm. Then these IMFs be passed as inputs to an ICA algorithm with the goal of estimated the source signals. Sequentially, reconstructing the new IMFs through multiplication between mixing matrix and IC components extracted without artifact, and the original signal can be obtained by summing remaining IMFs [91]. Figure 4 shows the general schematic of EMD-BSS method. The study of EEMD combined with ICA to remove EMG and ocular artifact from EEG was first present in [79]. The author also compared this algorithm with single-channel ICA and wavelet-ICA on real EEG signals. The results show that the EEMD-ICA algorithm has the best performance. Similar manner to EEMD-ICA, the EEMD-CCA [92-95], remove contaminated IMFs and then using unmixing matrix W, determined by CCA algorithm, to reconstruct source signals [96]. Another method utilized interchannel dependence information seen in few channel situation by combining multivariate empirical mode decomposition (MEMD) and CCA was discussed in [78]. Although it has been proven that MEMD-CCA is one of the most promising tool for muscle artifact removal under few-channel scenarios, the heavy computational cost limits it to be used in offline situation. 


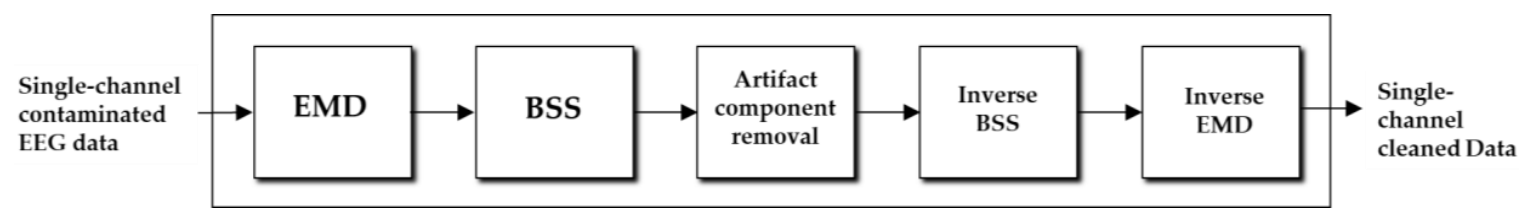

Figure 4. Process flow of EMD-BSS method.

\subsection{Wavelet-BSS}

ICA has crucial limitation that the number of sources need to be equal to the measurement sources, whilst WT fails to work when the artifacts overlap in the spectral domain, thus, a wavelet-ICA technique has been proposed in [97-100] to combine the positive portion to avoid shortcomings. Lin et al. [97] used WICA for single-channel artifact removal and Winkler et al. [99] removed contaminated EMG signals from EEG data. Firstly, the recorded EEG data is decomposed by wavelet transform and then the wavelet resolution contained probably artifactual components are fed into a chosen ICA algorithm. Finally the selecting components corrupted with artifacts removed and reconstruction of artifact-free EEG signals are performed using preserved wavelet components and disposed components. Figure 5 illustrates the process flow of Wavelet-BSS algorithms. Another commonly used version reversing the order of wavelet transform and ICA or CCA algorithm has been discussed in [101-104]. Other than ICA or CCA, wavelet can also be used with together PCA, as presented in the work of Kevric and Subasi $[105,106]$. In their method, wavelet analysis decompose the EEG signals into different frequency bands and then PCA is applied to obtain new coefficients of bands [107]. Conventional linear artifact removal methods usually smooth out the rapid jitter in the signal. The nonlinear filtering techniques Multiscale Principal Component Analysis (MSPCA) will preserve substantial amount of changes in the recordings and provide enhanced artifact removal performance than sole PCA algorithm [108].

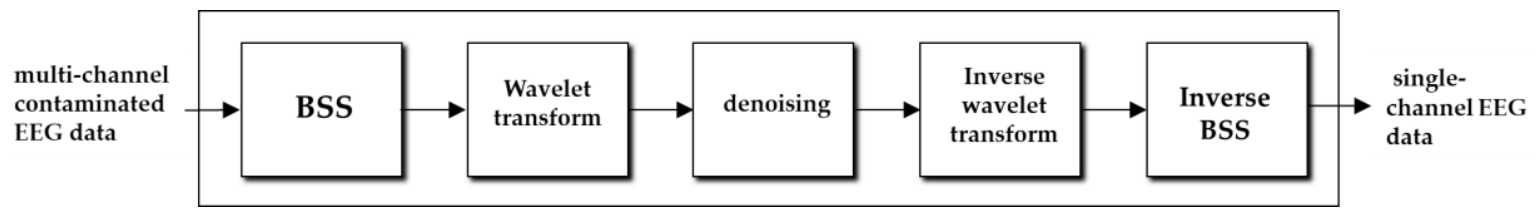

Figure 5. Process flow of BSS-WT method.

\subsection{BSS and Support Vector Machine}

Another hybrid method to extend the use of BSS is BSS-SVM. First, the recorded EEG data are decomposed into multiple components using BSS algorithms. Next, several features of components like temporal, spatial and statistical features are extracted. Then the features are used as input to a set of linear SVM classifier to identify artifact components. Finally, the remaining components are used to reconstructed artifact-free signals [21]. Figure 6 show the schematic of BSS-SVM method. Shoker et al. [109] first fused BBS and SVM to remove the eye-blinking artifacts. Halder et al. re-introduced this approach to classifier the artifactual components in EOG and EMG recording [110].

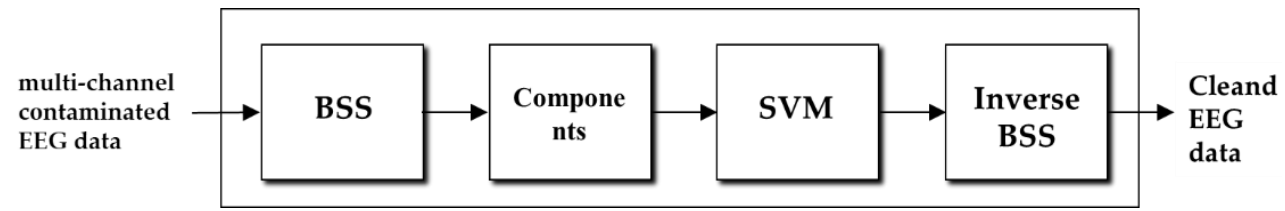

Figure 6. Process flow of BSS-SVM method. 


\section{Comparative Analysis}

The methods discussed above are among the most commonly used in EEG artifact removal. Some of these methods dealing with artifacts seed to minimize their presence by restricting eye movements and blinking during data collection or by excluding artifact-contaminated trials from the analyzed data.

Other methods seek to correct for ocular artifacts in the data, including regression in the time or frequency domain. Besides, others blindly separate artifacts from EEG signals. A detailed comparison between the mentioned techniques is provided in Table 2. There are a number of factors affect the choice of which algorithm to employ, which we discussed later.

Table 2. Comparative analysis of methods mentioned above.

\begin{tabular}{ccccc}
\hline Method & Additional Reference & Automatic & Online & Can Perform on Single Channel \\
\hline Regression & Y & Y & N & N \\
Wavelet & N & Y & N & Y \\
ICA & N & N & Y & N \\
CCA & N & Y & Y & Y \\
Adaptive filter & Y & Y & N & Y \\
Winner filter & N & Y & N & Y \\
Wavelet BSS & $\mathrm{N}$ & $\mathrm{N}$ & $\mathrm{N}$ & $\mathrm{N}$ \\
EMD BSS & $\mathrm{N}$ & $\mathrm{Y}$ & $\mathrm{Y}$ & \multicolumn{2}{c}{} \\
BSS-SVM & $\mathrm{N}$ & \multicolumn{2}{c}{}
\end{tabular}

Most of the EEG-based practical applications are often required real-time signal processing and can be robust to artifacts. This required the artifact removal methods implemented are capable of automatic and low computational cost. Automatic process means the chosen method can automatically identify and eliminate the artifact components without manual intervention. Regression and filtering approach can execute automatically when they have a reference signal. Moreover, BSS methods will be automatic when there is a subsequent procedure, as mentioned above, like SVM. Among these BSS methods, although ICA are the most commonly used techniques, the disregard of temporal or spatial relations within sources will result the loss of relevant information. But CCA algorithm can solve this problem and have a little computational time, which makes the algorithm applicable for real-time performance. Another factor which should be taken in account is the number of record channels. Especially for the home healthcare environment, the less of channel are often expected. BBS algorithms cannot be utilized in such situation, due to the principle of BSS that more channels will bring better accurate. But wavelet transform and EMD-based methods can executed with single channel since it can decompose a single records into multiple components [111]. However the reduction of measurement channel will cause the increase of computational complexity which will not be suitable for BCI and neurofeedback (NF) applications. In addition, the automatic methods are not usually general for artifact removal since there are multiple types of artifacts that are existed in the recordings. Hence the availability of reference signals will improve the accuracy and robustness of artifact removal by provide satisfactory complementary information. Also the information of the epochs of artifacts obtained by reference channel will reduce the computational cost. But the reference channel of each muscle contributing to EEG muscle artifacts are not feasible.

Apart from methods commented above, there are plenty of innovative and efficient approaches for artifact removal have been recently proposed. For instance, Corradino et al. [112] combined ICA with regression to automatically check, detection and clean residual artifacts. The technique presented to clean ICs can avoid the cut-band solutions. Recently, applied a multinomial regression classifier to establish a multiclass artifact identification system, which can automatically selected frequency, spatial and temporal features of the ICs. Considering that the trend of healthcare systems are developed toward few channels, Davies and James explored a single channel ICA method to decompose single channel signal into independent sources [113]. Another study developed the SASICA software plugin for the EEGLAB toolbox that encapsulating several artifact selection approach [114]. The interactive plots 
produced by this software guide human user to decide the removal of artifactual ICs. Daly et al. [115] proposed a method that combined wavelet decomposition, ICA and thresholding termed as FORCe. This online artifact removal method use for BCI applications. In 2016, a new method for real-time detection of eyeblink artifacts using an automatic thresholding algorithm was introduced [116]. Zou et al. extended the ICA method by the hierarchal clustering of IC features, which can identify both physiological and non-physiological artifacts form the EEG signals of BCI applications [117]. A recently emerging BSS algorithm which integrating the advantages of CCA and ICA into one single framework, independent vector analysis (IVA), remove muscle artifact by synchronously extracted the sources with maximal independence and maximal autocorrelation [118,119]. In addition, the combination of EEMD and IVA have been demonstrated that MEMD-IVA outperform other existing methods in a few channel situation [75]. More recently, a modified joint blind source separation (JBSS) approach and quadrature regression IVA (q-IVA) provide a more effective artifact removal technique in both time and frequency domain, paving the way for future research [120]. In addition, a filter-bank based artifacts removal approach was proposed by Dhindsa [121]. This method uses a supervise machine learning to detect artifacts by single channel, and outperforms Fully Automated Statistical Thresholding for EEG artifact Rejection (FASTER) due to its ability to identify small artifacts in the presence of high amplitude EEG. Mohammadpour et al. [122] made use of a Hidden Markov Model (HMM) machining learning method to remove eyeblink artifacts. Contrary to conventional algorithms, such machine learning based approaches that identify artifacts using huge datasets are tending to be a new research focus.

To conclude, ICA-based algorithms can deal with all kinds of artifact occurred in EEG recordings. Regression and adaptive filter are more feasible choices when the reference channels for specific artifacts are available. Apart from ICA, CCA and its combinations of other methods seem to be a good choice for removal of muscle artifacts. For application to a few channels, EMD, IVA, and its hybrid methods with BSS or WT could be an ideal choice. However, the requirement of a reference signal limits adaptive filter or regression methods to the removal of particular types of artifact. Wavelet transform fails to identify completely artifacts that overlap with spectral properties. EMD also suffers from the drawback of mode-mixing. Therefore, it is quite difficult to find a single method that is both efficient and accurate enough to satisfy all the conditions perfectly.

\section{Conclusions}

EEG signals are generated from the cerebral cortex and always be contaminated by some disturbances. In spite of the fact a number of techniques have been developed for removing undesired artifacts, an artifact removal method that combines high accuracy and algorithmic efficiency still needs to be identified. This paper summarized the primary techniques based on conclusions made in the published literatures. The advantages and the drawbacks of each method are also highlighted. Although most of the removal algorithms offer good performance, the methods listed above suffer from different limitations when utilized in a particular EEG-based application. Indeed, some methods are only focused on the detection and removal of particular artifacts, such as EOG, ECG, EMG. Some methods need reference channels to enhance the accuracy of artifact removal, which is not feasible of some specific applications. Some methods like BSS or Wavelet remove artifacts with great accuracy, however, methods operating with high computational complexity may not be suitable for online applications. Therefore, there is no optimal choice for remove all types of artifacts. Thus, one of the future objectives of the effective attenuation of artifacts is to develop an application-specific algorithm with better time and accuracy efficiency. Also in the current trend of artifacts removal, it can be concluded that the future directions will combine machine learning and traditional approaches for effective automatic artifact removal. Apart from that, new artifact removal algorithms for numerous types of artifacts in the multiple scenarios still need to be identified. 
Author Contributions: Conceptualization, G.-B.B. and X.J.; methodology, G.-B.B.; software, Z.T.; validation, G.-B.B., Z.T. and X.J.; formal analysis, X.J.; investigation, X.J., G.-B.B. and Z.T.; resources, G.-B.B.; data curation, X.J.; writing—original draft preparation, X.J., G.-B.B. and Z.T.; writing—review and editing, X.J., G.-B.B. and Z.T.; visualization, Z.T.; supervision, G.-B.B.; project administration, G.-B.B.; funding acquisition, G.-B.B.

Funding: This research was funded by National Natural Science Foundation of China (Grant U1713220), Beijing Natural Science Foundation (Grant 4161001) and Youth Innovation Promotion Association of the Chinese Academy of Sciences (Grant 2018165).

Conflicts of Interest: The authors declare no conflict of interest.

\section{References}

1. Frederik, V.; Luca, F.; Esin, K.; Jitkomut, S.; Pedro, A.V.; Daniele, M. Critical comments on EEG sensor space dynamical connectivity analysis. Brain Topogr. 2016, 1-12. [CrossRef]

2. Henry, J.C. Electroencephalography: Basic principles, clinical applications, and related fields, fifth edition. Neurology 2006, 67, 2092. [CrossRef]

3. Hirsch, L.J.; Brenner, R.P. Atlas of EEG in Critical Care; John Wiley and Sons: Hoboken, NJ, USA, 2010; Volume 30, pp. 187-216, ISBN 9780470746707.

4. Nunez, P.L.; Srinivasan, R. Electric Fields of the Brain: The Neurophysics of EEG, 2nd ed.; Oxford University Press: New York, NY, USA, 2005; pp. 154-169, ISBN 9780195050387.

5. Wang, H.; Lei, X.; Zhan, Z.; Yao, L.; Wu, X. A new fMRI informed mixed-norm constrained algorithm for EEG source localization. IEEE Access 2018, 6, 8258-8269. [CrossRef]

6. Radüntz, T.; Scouten, J.; Hochmuth, O.; Meffert, B. Automated EEG artifact elimination by applying machine learning algorithms to ICA-based features. J. Neural Eng. 2017, 14, 8-15. [CrossRef] [PubMed]

7. Ge, S.; Yang, Q.; Wang, R.; Lin, P.; Gao, J.; Leng, Y.; Yang, Y.; Wang, H. A brain-computer interface based on a few-channel EEG-fNIRS bimodal system. IEEE Access 2017, 5, 208-218. [CrossRef]

8. Fatourechi, F.; Bashashati, A.; Ward, R.K.; Birch, G.E. EMG and EOG artifacts in brain computer interface systems: A survey. Clin. Neurophysiol. 2007, 118, 480-494. [CrossRef] [PubMed]

9. Naeem Mannan, M.; Ahmad, K.M.; Shinil, K.; Myung, Y.J. Effect of EOG Signal Filtering on the Removal of Ocular Artifacts and EEG-Based Brain-Computer Interface: A Comprehensive Study. Complexity 2018, 2018, 18-36. [CrossRef]

10. Tamburro, G.; Fiedler, P.; Stone, D.; Haueisen, J.; Comani, S. A new ICA-based fingerprint method for the automatic removal of physiological artifacts from EEG recordings. Peer] 2018, 6. [CrossRef] [PubMed]

11. Labate, D.; La Foresta, F.; Mammone, N.; Morabito, F.C. Effects of Artifacts Rejection on EEG Complexity in Alzheimer's Disease. In Advances in Neural Networks: Computational and Theoretical Issues; Bassis, S., Esposito, A., Morabito, F., Eds.; Springer: Cham, Switzerland, 2015; Volume 37, pp. 129-136, ISBN 978-3-319-18163-9.

12. Husseen, A.H.; Emmanuel, J.; Sun, L.; Emmanuel, I. Complexity Measures for Quantifying Changes in Electroencephalogram in Alzheimer s Disease. Complexity 2018, 2018, 1-12. [CrossRef]

13. Sweeney, K.T.; Ward, T.E.; McLoone, S.F. Artifact removal in physiological signals practices and possibilities. IEEE Trans. Inf. Technol. Biomed. 2012, 16, 488-500. [CrossRef] [PubMed]

14. Somers, B.; Bertrand, A. Removal of eye blink artifacts in wireless EEG sensor networks using reduced-bandwidth canonical correlation analysis. J. Neural Eng. 2016, 13, 066008. [CrossRef] [PubMed]

15. Teixeira, A.; Tome, A.; Lang, E.; Gruber, P.; Martins da Silva, A. Automatic removal of high-amplitude artefacts from single-channel electroencephalograms. Comput. Methods Programs Biomed. 2006, 83, 125-138. [CrossRef] [PubMed]

16. James, C.J.; Hesse, C.W. Independent component analysis for biomedical signals. Physiol. Meas. 2005, 26, 15-39. [CrossRef]

17. Niedermeyer, E.; Lopes da Silva, F.H. Electroencephalography: Basic Principles, Clinical Applications, and Related Fields, 5th ed.; Raven Press: New York, NY, USA, 2005; pp. 654-660, ISBN 978-0781789424.

18. Minguillon, J.; Lopez-Gordo, M.A.; Pelayo, F. Trends in EEG-BCI for daily-life: Requirements for artifact removal. Biomed. Signal Process. Control 2017, 31, 407-418. [CrossRef]

19. Johal, P.K.; Jain, N. Artifact removal from EEG: A comparison of techniques. In Proceedings of the 2016 International Conference on Electrical, Electronics, and Optimization Techniques, Chennai, Indian, 3-5 March 2016. 
20. Jebelli, H.; Hwang, S.; Lee, S. EEG-based workers' stress recognition at construction sites. Autom. Constr. 2018, 93, 315-324. [CrossRef]

21. Urigüen, J.A.; Garciazapirain, B. EEG artifact removal—State-of-the-art and guidelines. J. Neural Eng. 2015, 12. [CrossRef] [PubMed]

22. Huppert, T.J.; Diamond, S.G.; Franceschini, M.A.; Boas, D.A. HomER: A review of time-series analysis methods for near-infrared spectroscopy of the brain. Appl. Opt. 2009, 48, D280-D298. [CrossRef] [PubMed]

23. Islam, M.K.; Rastegarnia, A.; Yang, Z. Methods for Artifact Detection and Removal from Scalp EEG: A Review. Clin. Neurophysiol. 2016, 46, 287-385. [CrossRef] [PubMed]

24. Anderer, P.; Roberts, S.; Schlögl, A.; Gruber, G.; Klösch, G.; Herrmann, W.; Rappelsberger, P.; Filz, O.; Barbanoj, M.J.; Dorffner, G.; et al. Artifact processing in computerized analysis of sleep EEG-A review. Neuropsychobiology 1999, 40, 150-157. [CrossRef] [PubMed]

25. Garrick, L.W.; Robert, E.K.; Anita, M.; Jeffrey, F.C.; Nathan, A.F. Automatic correction of ocular artifacts in the EEG: A comparison of regression-based and component-based methods. Int. J. Psychophysiol. 2004, 53, 105-119. [CrossRef]

26. Schlögl, A.; Keinrath, C.; Zimmermann, D.; Scherer, R.; Leeb, R.; Pfurtscheller, G. A fully automated correction method of EOG artifacts in EEG recordings. Clin. Neurophysiol. 2007, 118, 98-104. [CrossRef] [PubMed]

27. Hamal, A.Q.; Rehman, A.W.B.A. Artifact Processing of Epileptic EEG Signals: An Overview of Different Types of Artifacts. In Proceedings of the 2013 International Conference on Advanced Computer Science Applications and Technologies, Kuching, Malaysia, 22-24 December 2013.

28. Croft, R.J.; Barry, R.J. Removal of ocular artifacts from the EEG: A review. Clin. Neurophysiol. Clin. 2000, 30, 5-19. [CrossRef]

29. Goncharova, I.I.; Mcfarland, D.J.; Vaughan, T.M.; Wolpaw, J.R. EMG contamination of EEG: Spectral and topographical characteristics. Clin. Neurophysiol. 2003, 114, 1580-1593. [CrossRef]

30. Mcmenamin, B.W.; Shackman, A.J.; Greischar, L.L.; Davidson, R.J. Electromyogenic artifacts and electroencephalographic inferences revisited. Neuroimage 2011, 54, 4-9. [CrossRef] [PubMed]

31. Devuyst, S.; Dutoit, T.; Stenuit, P.; Kerkhofs, M.; Stanus, E. Removal of ECG artifacts from EEG using a modified independent component analysis approach. In Proceedings of the 2008 30th Annual International Conference of the IEEE Engineering in Medicine and Biology Society, Vancouver, BC, Canada, 20-25 August 2008.

32. Chen, X.; Liu, A.; Chiang, J.; Wang, Z.J.; Mckeown, M.J.; Ward, R.K. Removing muscle artifacts from EEG data: Multichannel or single-channel techniques? IEEE Sens. J. 2016, 16, 1986-1997. [CrossRef]

33. Lee, K.J.; Park, C.; Lee, B. Elimination of ECG Artifacts from a Single-Channel EEG Using Sparse Derivative Method. In Proceedings of the 2015 IEEE International Conference on Systems, Man, and Cybernetics, Kowloon, China, 9-12 Octorber 2015.

34. Nolte, G.; Bai, O.; Wheaton, L.; Mari, Z.; Vorbach, S.; Hallett, M. Identifying true brain interaction from EEG data using the imaginary part of coherency. Clin. Neurophysiol. 2014, 115, 2292-2307. [CrossRef] [PubMed]

35. Qin, Y.; Xu, P.; Yao, D. A comparative study of different references for EEG default mode network: The use of the infinity reference. Clin. Neurophysiol. 2010, 121, 1981-1991. [CrossRef] [PubMed]

36. Dong, L.; Li, F.; Liu, Q.; Wen, X.; Lai, Y.; Xu, P.; Yao, D. Matlab toolboxes for reference electrode standardization technique (REST) of scalp EEG. Front. Neurosci. 2017, 11, 601-608. [CrossRef] [PubMed]

37. Klados, M.A.; Papadelis, C.; Braun, C.; Bamidis, P.D. REG-ICA: A hybrid methodology combining blind source separation and regression techniques for the rejection of ocular artifacts. Biomed. Signal Process Control 2011, 10, 291-300. [CrossRef]

38. Hillyard, S.A.; Galambos, R. Eye movement artifact in the CNV. Electroencephalogr. Neurophysiol. 1970, 28, 173-182. [CrossRef]

39. Whitton, J.L.; Lue, F.; Moldofsky, H. A spectral method for removing eye movement artifacts from the EEG. Electroencephalogr. Clin. Neurophysiol. 1978, 44, 735-741. [CrossRef]

40. Wallstrom, G.L.; Kass, R.E.; Miller, A.; Cohn, J.; Fox, N. Correction of ocular artifacts in the EEG using Bayesian adaptive regression splines. In Case Studies in Bayesian Statistics; Gatsonis, C., Kass, R.E., Carriquiry, A., Gelman, A., Higdon, D., Pauler, D.K., Verdinelli, I., Eds.; Springer: New York, NY, USA, 2002; Volume 6, pp. 351-356, ISBN 978-0-387-95472-1. 
41. Flumeri, G.D.; Aricó, P.; Borghini, G.; Colosimo, A.; Babiloni, F. A New Regression-based Method for the Eye Blinks Artifacts Correction in the EEG Signal, without Using Any EOG Channel. In Proceedings of the 2016 38th Annual International Conference of the IEEE Engineering in Medicine and Biology Society (EMBC), Orlando, FL, USA, 16-20 August 2016.

42. Kumar, S.P.; Arumuganathan, R.; Sivakuma, K.; Vimal, C. Removal of Ocular Artifacts in the EEG through Wavelet Transform without using an EOG Reference Channel. Int. J. Open Probl. Comput. Math. 2008, 1, 189-200.

43. Safieddine, D.; Kachenoura, A.; Albera, L.; Birot, G.; Karfoul, A.; Pasniu, A. Removal of muscle artifact from EEG data: Comparison between stochastic (ICA and CCA) and deterministic (EMD and wavelet-based) approaches. EURASIP J. Adv. Signal Process. 2012, 2012. [CrossRef]

44. Lakshmi, K.G.A.; Surling, S.N.N.; Sheeba, O. A Novel Approach for the Removal of Artifacts in EEG Signals. In Proceedings of the 2017 International Conference on Wireless Communications, Signal Processing and Networking (WiSPNET), Chennai, India, 22-24 March 2017.

45. Berg, P.; Scherg, M. Dipole modeling of eye activity and its application to the removal of eye artefacts from the EEG ad MEG. Clin. Phys. Physiol. Meas. 1991, 12, 49-54. [CrossRef] [PubMed]

46. Casarotto, S.; Bianchi, A.M.; Cerutti, S.; Chiarenza, G.A. Principal component analysis for reduction of ocular artefacts in event-related potentials of normal and dyslexic children. Clin. Neurophysiol. 2004, 115, 609-619. [CrossRef] [PubMed]

47. Jung, T.P.; Makieg, S.; Bell, A.J.; Sejnowski, T.J. Independent component analysis of electroencephalographic and event-related potential data. Cent. Audit. Process. Neural Model. 1996, 2, 1548-1551.

48. Vigário, R. Extraction of ocular artifacts from EEG using independent component analysis. Electroencephalogr. Clin. Neurophysiol. 1997, 103, 395-404. [CrossRef]

49. Vigário, R.; Särelä, J.; Jousmäki, V.; Hämäläinen, M.; Oja, E. Independent component approach to the analysis of EEG and MEG recordings. IEEE Trans. Biomed. Eng. 2000, 47, 589-593. [CrossRef] [PubMed]

50. Jung, T.P.; Makeig, S.; Westerfield, M.; Townsend, J.; Courchesne, E.; Sejnowski, T.J. Removal of eye activity artifacts from visual event-related potentials in normal and clinical subjects. Clin. Neurophysiol. 2000, 111, 1745-1758. [CrossRef]

51. Romero, S.; Mailanas, M.; Clos, S.; Gimenez, S.; Barbanoj, M.J. Reduction of EEG Artifacts by ICA in Different Sleep Stages. In Proceedings of the 25th Annual International Conference of the IEEE Engineering in Medicine and Biology Society, Cancun, Mexico, 17-21 September 2003.

52. Delorme, A.; Makeig, S.; Sejnowski, T. Automatic artifact rejection for EEG data using high-order statistics and independent component analysis. In Proceedings of the Third International ICA Conference, San Diego, CA, USA, 9-12 December 2001.

53. Joyce, C.A.; Gorodnitsky, I.F.; Kutas, M. Automatic removal of eye movement and blink artifacts from EEG data using blind component separation. Psychophysiology 2004, 41, 313-325. [CrossRef] [PubMed]

54. Bian, N.Y.; Wang, B.; Cao, Y.; Zhang, L. Automatic Removal of Artifacts from EEG Data Using ICA and Exponential Analysis. In Proceedings of the Third International Conference on Advances in Neural Networks, Chengdu, China, 28 May-1 June 2006.

55. Li, Y.; Ma, Z.; Lu, W.; Li, Y. Automatic removal of the eye blink artifact from EEG using an ICA based template matching approach. Physiol. Meas. 2006, 27, 425-436. [CrossRef] [PubMed]

56. Flexer, A.; Bauer, H.; Pripfl, J.; Dorffner, G. Using ICA for removal of ocular artifacts in EEG recorded from blind subjects. Neural Netw. 2005, 18, 998-1005. [CrossRef] [PubMed]

57. Ting, K.H.; Fung, P.C.W.; Chang, C.Q.; Chan, F.H.Y. Automatic correction of artifact from single trial event-related potentials by blind source separation using second order statistics only. Med. Eng. Phys. 2006, 28, 780-794. [CrossRef] [PubMed]

58. Zhou, W.; Gotman, J. Automatic removal of eye movement artifacts from the EEG using ICA and the dipole model. Prog. Nat. Sci. 2009, 19, 1165-1170. [CrossRef]

59. Frølich, L.; Dowding, I. Removal of muscular artifacts in EEG signals: A comparison of linear decomposition methods. Brain Inform. 2018, 5, 13-22. [CrossRef] [PubMed]

60. Jung, T.P.; Humphries, C.; Lee, T.W.; Makeig, S.; Mckeown, M.J.; Iragui, V.; Sejnowski, T.J. Extended ICA Removes Artifacts from Electroencephalographic Recordings. In Advances in Neural Information Processing Systems; MIT Press Ltd.: Denver, CO, USA, 1998. 
61. Vayá, C.; Rieta, J.J.; Sánchez, C.; Moratal, D. Convolutive blind source separation algorithms applied to the electrocardiogram of atrial fibrillation: Study of performance. IEEE Trans. Biomed. Eng. 2007, 54, 1530-1533. [CrossRef] [PubMed]

62. Borga, M.; Friman, O.; Lundberg, P.; Knutsson, H. A Canonical Correlation Approach to Exploratory Data Analysis in fMRI. In Proceedings of the ISMRM 10th Scientific Meeting \& Exhibition, Honolulu, HI, USA, 18-24 May 2002.

63. Dong, L.; Zhang, Y.; Zhang, R.; Zhang, X.; Gong, D.; Valdes-Sosa, P.A.; Xu, P.; Luo, C.; Yao, D. Characterizing nonlinear relationships in functional imaging data using eigenspace maximal information canonical correlation analysis (emiCCA). Neuroimage 2015, 109, 388-401. [CrossRef] [PubMed]

64. Clercq, W.D.; Vergult, A.; Vanrumste, B.; Paesschen, W.V.; Huffel, S.V. Canonical correlation analysis applied to remove muscle artifacts from the electroencephalogram. IEEE Trans. Biomed. Eng. 2006, 53, 2583-2587. [CrossRef] [PubMed]

65. Vos, D.; Riècvs, S.; Vanderperren, K.; Vanrumste, B.; Alario, F.X.; Van, H.S. Removal of muscle artifacts from EEG recordings of spoken language production. Neuroinformatics 2010, 8, 135-150. [CrossRef] [PubMed]

66. Kaiboriboon, K.; Hans, O.; Lüders, H.M.; Turnbull, J.; Lhatoo, S.D. EEG source imaging in epilepsy-Practicalities and pitfalls. Nat. Rev. Neurol. 2012, 8, 498-507. [CrossRef] [PubMed]

67. Gorodnitsky, I.F.; George, J.S.; Rao, B.D. Neuromagnetic source imaging with focuss: A recursive weighted minimum norm algorithm. Electroencephalogr. Clin. Neurophysiol. 1995, 95, 231-251. [CrossRef]

68. Liu, T.; Yao, D. Removal of the ocular artifacts from EEG data using a cascaded spatio-temporal processing. Comput. Methods Programs Biomed. 2006, 83, 95-103. [CrossRef] [PubMed]

69. Yao, D. A method to standardize a reference of scalp EEG recordings to a point at infinity. Physiol. Meas. 2001, 22, 693-711. [CrossRef] [PubMed]

70. Huang, N.E.; Shen, Z.; Long, S.R.; Wu, M.C.; Shih, H.H.; Zheng, Q.; Yen, N.C.; Tung, C.C.; Liu, H.H. The empirical mode decomposition and the hilbert spectrum for nonlinear and non-stationary time series analysis. Proc. Math. Phys. Eng. Sci. 1998, 454, 903-995. [CrossRef]

71. Molla, M.K.I.; Islam, M.R.; Tanaka, T.; Rutkowski, T.M. Artifact suppression from EEG signals using data adaptive time domain filtering. Neurocomputing 2012, 97, 297-308. [CrossRef]

72. Chavez, M.; Grosselin, F.; Bussalb, A.; Fallani, F.D.V.; Navarro-Sune, X. Surrogate-based artifact removal from single channel EEG. IEEE Trans. Neural Syst. Rehabil. Eng. 2018, 26, 540-550. [CrossRef] [PubMed]

73. Yang, B.; Zhang, T.; Zhang, Y.; Liu, W.; Wang, J.; Duan, K. Removal of electrooculogram artifacts from electroencephalogram using canonical correlation analysis with ensemble empirical mode decomposition. Cogn. Comput. 2017, 9, 626-633. [CrossRef]

74. Zhang, Y.; Xu, P.; Li, P.; Duan, K.; Wen, Y.; Yang, Q.; Zhang, T.; Yao, D. Noise-assisted multivariate empirical mode decomposition for multichannel EMG signals. Biomed. Eng. Online 2017, 16, 107-123. [CrossRef] [PubMed]

75. Xu, X.; Chen, X.; Zhang, Y. Removal of muscle artefacts from few-channel EEG recordings based on multivariate empirical mode decomposition and independent vector analysis. Electron. Lett. 2018, 54, 866-868. [CrossRef]

76. Wu, Z.; Huang, N.E. Ensemble empirical mode decomposition: A noise-assisted data analysis method. Adv. Adapt. Data Anal. 2011, 1, 1-41. [CrossRef]

77. Mijovi, C.B.; Vos, M.D.; Gligorijevic, I.; Taelman, J.; Van Huffel, S. Source separation from single channel recordings by combining empirical mode decomposition and independent component analysis. IEEE Trans. Biomed. Eng. 2010, 57, 2188-2196. [CrossRef] [PubMed]

78. Chen, X.; Xu, X.; Liu, A.; Mckeown, M.J.; Wang, Z.J. The use of multivariate EMD and CCA for denoising muscle artifacts from few-channel EEG recordings. IEEE Trans. Instrum. Meas. 2018, 67, 359-370. [CrossRef]

79. He, P.; Wilson, G.; Russell, C.; Gerschutz, M. Removal of Ocular Artifacts from EEG: A Comparison of Adaptive Filtering Method and Regression Method Using Simulated Data. In Proceedings of the IEEE 27th Annual Conference on Engineering in Medicine and Biology, Shanghai, China, 17-18 January 2006.

80. Marque, C.; Bisch, C.; Dantas, R.; Elayoubi, S.; Brosse, V.P.; Erot, C. Adaptive filtering for ECG rejection from surface EMG recordings. J. Electromyogr. Kinesiol. 2005, 15, 310-315. [CrossRef] [PubMed]

81. He, P.; Wilson, G.; Russell, C. Removal of ocular artifacts from electro-encephalogram by adaptive filtering. Med. Biol. Eng. Comput. 2004, 42, 407-412. [CrossRef] [PubMed] 
82. Kher, R.; Gandhi, R. Adaptive Filtering Based Artifact Removal from Electroencephalogram (EEG) Signals. In Proceedings of the 2016 International Conference on Communication and Signal Processing (ICCSP), Melmaruvathur, India, 6-8 April 2016.

83. Somers, B.; Francart, T.; Bertrand, A. A generic EEG artifact removal algorithm based on the multi-channel Wiener filter. J. Neural Eng. 2018, 15. [CrossRef] [PubMed]

84. Izzetoglu, M.; Devaraj, A.; Bunce, S.; Onaral, B. Motion artifact cancellation in NIR spectroscopy using Wiener filtering. IEEE Trans. Biomed. Eng. 2005, 52, 934-938. [CrossRef] [PubMed]

85. Donoho, D.L. Sparse components of images and optimal atomic decompositions. Constr. Approx. 2001, 17, 353-382. [CrossRef]

86. Silva, A.R.F.D. Atomic decomposition with evolutionary pursuit. Digit. Signal Process. 2003, 13, $317-337$. [CrossRef]

87. Mallat, S.G.; Zhang, Z. Matching pursuits with time-frequency dictionaries. IEEE Trans. Signal Process. 1993, 41, 3397-3415. [CrossRef]

88. Xu, P.; Yao, D. Two dictionaries matching pursuit for sparse decomposition of signals. Signal Process. 2006, 86, 3472-3480. [CrossRef]

89. Li, P.; Xu, P.; Zhang, R.; Guo, L.; Yao, D. L1 Norm based common spatial patterns decomposition for scalp EEG BCI. Biomed. Eng. Online 2013, 12,77-88. [CrossRef] [PubMed]

90. Li, P.; Wang, X.; Li, F.; Zhang, R.; Ma, T.; Peng, Y.; Lei, X.; Tian, Y.; Guo, D.; Liu, T.; et al. Autoregressive model in the Lp norm space for EEG analysis. J. Neurosci. Methods 2014, 240, 170-178. [CrossRef] [PubMed]

91. Chen, X.; Wang, Z.J.; Mckeown, M. Joint blind source separation for neurophysiological data analysis: Multiset and multimodal methods. IEEE Signal Process. Mag. 2016, 33, 86-107. [CrossRef]

92. Chen, X.; Chen, Q.; Zhang, Y.; Wang, Z.J. A novel EEMD-CCA approach to removing muscle artifacts for pervasive EEG. IEEE Sens. J. 2018, 99. [CrossRef]

93. Chen, X.; He, C.; Peng, H. Removal of muscle artifacts from single-channel EEG based on ensemble empirical mode decomposition and multiset canonical correlation analysis. J. Appl. Math. 2014, 2014. [CrossRef]

94. Sweeney, K.T.; McLoone, S.F.; Ward, T.E. The use of ensemble empirical mode decomposition with canonical correlation analysis as a novel artifact removal technique. IEEE Trans. Biomed. Eng. 2013, 60, 97-105. [CrossRef] [PubMed]

95. Wang, G.; Teng, C.; Li, K.; Zhang, Z.; Yan, X. The removal of EOG artifacts from EEG signals using independent component analysis and multivariate empirical mode decomposition. IEEE J. Biomed. Health Inf. 2016, 20, 1301-1308. [CrossRef] [PubMed]

96. Soomro, M.H.; Badruddin, N.; Yusoff, M.Z.; Jatoi, M.A. Automatic Eye-blink Artifact Removal Method Based on EMD-CCA. In Proceedings of the 2013 ICME International Conference on Complex Medical Engineering, Beijing, China, 25-28 May 2013.

97. Lin, J.; Zhang, A. Fault feature separation using wavelet-ICA filter. NDT E Int. 2005, 38, 421-427. [CrossRef]

98. Azzerboni, B.; Carpentieri, M.; Foresta, F.L.; Morabito, F.C. Neural-ICA and Wavelet Transform for Artifacts Removal in Surface EMG. In Proceedings of the 2004 IEEE International Joint Conference on Neural Networks, Budapest, Hungary, 25-29 July 2004.

99. Winkler, I.; Brandl, S.; Horn, F.; Waldburger, E.; Allefeld, C.; Tangermann, M. Robust artifactual independent component classification for BCI practitioners. J. Neural Eng. 2014, 11. [CrossRef] [PubMed]

100. Calcagno, S.; Foresta, F.; Versaci, M. Independent component analysis and discrete wavelet transform for artifact removal in biomedical signal processing. Am. J. Appl. Sci. 2014, 11, 57-68. [CrossRef]

101. Mammone, N.; Morabito, F. Enhanced automatic wavelet independent component analysis for electroencephalographic artifact removal. Entropy 2014, 16, 6553-6572. [CrossRef]

102. Castellanos, N.P.; Makarov, V.A. Recovering EEG brain signals: Artifact suppression with wavelet enhanced independent component analysis. J. Neurosci. Methods 2006, 158, 300-312. [CrossRef] [PubMed]

103. Hamaneh, M.B.; Chitravas, N.; Kaiboriboon, K.; Lhatoo, S.D.; Loparo, K.A. Automated removal of EKG artifact from EEG data using independent component analysis and continuous wavelet transformation. IEEE Trans. Biomed. Eng. 2014, 61, 1634-1641. [CrossRef] [PubMed]

104. Mahajan, R.; Morshed, B.I. Unsupervised eye blink artifact denoising of EEG data with modified multiscale sample entropy, kurtosis, and wavelet-ICA. IEEE J. Biomed. Health Inform. 2015, 19, 158-165. [CrossRef] [PubMed] 
105. Kevric, J.; Subasi, A. The effect of multiscale PCA de-noising in epileptic seizure detection. J. Med. Syst. 2014, 38, 131-289. [CrossRef] [PubMed]

106. Kevric, J.; Subasi, A. The impact of Mspca signal de-noising in real-time wireless brain computer interface system. Southeast Eur. J. Soft Comput. 2015, 4, 43-47. [CrossRef]

107. Alickovic, E.; Subasi, A. Ensemble SVM method for automatic sleep stage classification. IEEE Trans. Instrum. Meas. 2018, 67, 1258-1265. [CrossRef]

108. Kevric, J.; Subasi, A. Comparison of signal decomposition methods in classification of EEG signals for motor-imagery BCI system. Biomed. Signal Process. Control 2017, 31, 398-406. [CrossRef]

109. Shoker, L.; Sanei, S.; Chambers, J. Artifact removal from electroencephalograms using a hybrid BSS-SVM algorithm. IEEE Signal Process. Lett. 2005, 12, 721-724. [CrossRef]

110. Halder, S.; Bensch, M.; Bogdan, M.; Birbaumer, N.; Rosenstiel, W. Online artifact removal for brain-computer interfaces using support vector machines and blind source separation. Comput. Intell. Neurosci. 2007, 2007. [CrossRef] [PubMed]

111. Chen, X.; Liu, A.; Peng, H.; Ward, R. A preliminary study of muscular artifact cancellation in single-channel EEG. Sensors 2014, 14, 18370-18389. [CrossRef] [PubMed]

112. Corradino, C.; Bucolo, M. Automatic preprocessing of EEG signals in long time scale. In Proceedings of the 2015 37th Annual International Conference of the IEEE Engineering in Medicine and Biology Society (EMBC), Milan, Italy, 25-29 August 2015.

113. Davies, M.E.; James, C.J. Source separation using single channel ICA. Signal Process. 2007, 87, $1819-1832$. [CrossRef]

114. Castro-Puyana, M.; García-Ruiz, C.; Cifuentes, A.; Crego, A.L.; Marina, M.L. A practical guide to the selection of independent components of the electroencephalogram for artifact correction. J. Neurosci. Methods 2015, 250, 47-63. [CrossRef]

115. Daly, I.; Scherer, R.; Billinger, M.; Müller-Putz, G. FORCe: Fully online and automated artefact removal for brain-computer interfacing. IEEE Trans. Neural Syst. Rehabil. Eng. 2015, 23, 725-736. [CrossRef] [PubMed]

116. Chang, W.D.; Lim, J.H.; Im, C.H. An unsupervised eye blink artefact detection method for real-time electroencephalogram processing. Physiol. Meas. 2016, 37, 401-417. [CrossRef] [PubMed]

117. Zou, Y.; Nathan, V.; Jafari, R. Automatic identification of artefact-related independent components for artefact removal in EEG recordings. IEEE J. Biomed. Health Inform. 2016, 20, 73-81. [CrossRef] [PubMed]

118. Chen, X.; Peng, H.; Yu, F.; Wang, K. Independent vector analysis applied to remove muscle artifacts in EEG data. IEEE Trans. Instrum. Meas. 2017, 66, 1770-1779. [CrossRef]

119. Chen, X.; Liu, A.; Chen, Q.; Liu, Y.; Zou, L.; Mckeown, M.J. Simultaneous ocular and muscle artifact removal from EEG data by exploiting diverse statistics. Comput. Biol. Med. 2017, 88, 1-10. [CrossRef] [PubMed]

120. Lee, S.; McKeown, M.J.; Wang, Z.J.; Chen, X. Removal of high-voltage brain stimulation artifacts from simultaneous EEG recordings. IEEE Trans. Biomed. Eng. 2019, 66, 50-60. [CrossRef] [PubMed]

121. Dhindsa, K. Filter-bank artifact rejection: High performance real-time single-channel artifact detection for EEG. Biomed. Signal Process. Control 2017, 38, 224-235. [CrossRef]

122. Mohammadpour, M.; Rahmani, V. A Hidden Markov Model-based approach to removing EEG artifact. Fuzzy Intell. Syst. IEEE 2017, 46-49. [CrossRef]

(C) 2019 by the authors. Licensee MDPI, Basel, Switzerland. This article is an open access article distributed under the terms and conditions of the Creative Commons Attribution (CC BY) license (http:/ / creativecommons.org/licenses/by/4.0/). 